\title{
Drug Closure of a Patent Ductus Arteriosus in an Extremely Low Birth Weight Premature Newborn. A Case Report
}

\author{
Elena Moldovan ${ }^{1 *}$, Manuela Cucerea ${ }^{1,2}$ \\ 1 University of Medicine and Pharmacy Tîrgu-Mureş, Romania, 38 Gheorghe Marinescu street, Tirgu Mures, 540139, \\ Romania \\ 2 Regional Center of Neonatal Intensive Care Unit UGON, 50 Gheorghe Marinescu street, Tirgu Mures, 540139, \\ Romania
}

\begin{abstract}
Introduction: Patent ductus arteriosus involves maintaining the permeability of the vascular ductus located between the pulmonary artery and the descending aorta, due to the failure of transition from foetal to adult type circulation. This malformation is characteristic to premature newborns with extremely low birth weight. The main pathophysiological factors identified in this pathology are immaturity of the smooth muscles, presence of vasodilator mediators and persistent hypoxaemia. Ductal-dependent cardiac malformations require drug therapy for keeping the permeability of the ductus arteriosus until the time of corrective surgery.
\end{abstract}

Case presentation: We present the case of an extremely low birth weight premature newborn, derived from twin pregnancy with suspected specific pathology, respectively feto-fetal transfusion syndrome, admitted to the Regional Centre of Neonatal Intensive Care Unit Tîrgu-Mureş.

Premature newborn with gestational age 31-32 weeks, extremely low birth weight, derived from twin pregnancy, with adequate prenatal follow up, was born by caesarean section for severe intrauterine growth restriction secondary to feto-fetal transfusion syndrome suspicion. The newborn developed respiratory distress syndrome by surfactant deficiency, intraventricular-haemorrhage grade I/II, and severe retinopathy. The detection of a systolic murmur in the second week of life, the diastolic theft revealed by trans-fontanellar ultrasound as well as lowered diastolic blood pressure values raised the suspicion of a patent ductus arteriosus and therefore specific treatment with ibuprofen, a cyclooxygenase inhibitor, was initiated. Progressive alteration of the newborn's condition and the evidence of a coarctation of the aorta imposed the initiation of Prostaglandin E1 therapy and subsequent surgical correction.

Conclusions: Although beneficial, prophylactic or therapeutic closure of persistent ductus arteriosus may worsen the evolution of a newborn with a "silent" cardiac malformation and associated pathology.

Keywords: extremely low birth weight, patent ductus arteriosus, feto-fetal transfusion syndrome

Received: 09 December 2014 / Accepted: 15 January 2015

\section{INTRODUCTION}

Prematurity and congenital heart diseases represent the main causes of morbidity and mortality in the neonatal intensive care centres [1]. An extremely low birth-weight premature new-born has a gestational age below 37 weeks and weight less than 1000 grams. The remarkable progress achieved in neonatal intensive care, the increasing rate of adequate prenatal followup visits, as well as the identification of patients at risk of preterm birth, led to a decrease in neonatal mortality rate among extremely low birth weight premature newborns. The use of surfactant in the prevention and treatment of respiratory distress syndrome significantly contributes to the increasing rate of survival among premature newborns, nonetheless associated late complications, such as cognitive deficits, cerebral palsy or sensorineural disorders remain ever present $[2,3]$. Patent ductus arteriosus involves maintaining the permeability of the vascular ductus located between the pul-

\footnotetext{
* Correspondence to: Elena Moldovan, University of Medicine and Pharmacy of Tirgu Mures, 38 Gheorghe Marinescu street, Tirgu Mures, 540139, Romania. Email: bitirelena@gmail.com
} 
monary artery and the descending aorta, due to the failure of transition from foetal to adult type circulation. Physiologically, constriction induced by the increase in oxygen concentration, determines the closure of the ductus arteriosus within the first 48 hours of life. The main pathophysiological factors identified in this pathology are immaturity of the smooth muscles, manifested by the contractility lack of the vascular duct following the increase in oxygen concentration, presence of vasodilator mediators and persistent hypoxaemia, commonly found in premature newborns with an associated pulmonary pathology. Chromosomal abnormalities, rubella infection in the first trimester, foetal alcohol syndrome, maternal consumption of amphetamines as well as the use of phenytoin during pregnancy, represent the main identified aetiological factors [4]. Patent ductus arteriosus is a common pathology in extremely low birth weight premature newborns, being identified in $80 \%$ of cases. This increases the risk of developing intraventricular haemorrhage, necrotic ulcerative enterocolitis, or chronic lung disease [5]. Clinical diagnosis of patent ductus arteriosus in the premature new-born weighing less than 1000 grams represents a challenge, given the association with respiratory distress syndrome by surfactant deficiency [3]. The new-born weighing less than 1200 grams has difficulties in tolerating the persistent ductus arteriosus, compared to the mature new-born, bearing in mind the specific pathology of impaired alveoli development and surfactant deficiency associated with this category of patients [3]. The classic clinical aspect of this malformation appears only after the involution of the respiratory distress syndrome caused by surfactant deficiency [6]. The optimum time for closing the patent ductus arteriosus in premature newborns is unknown [7]. Prophylactic closure therapy of the patent ductus arteriosus in the new-born weighing less than 1000 grams, in the first three days of life, leads to a significant decrease in the need for surgical ligation, decrease in the need of ventilator support, and faster regain of the birth weight [8]. Although the use of cyclooxygenase inhibitors has the same therapeutic efficacy, it is shown that ibuprofen results in fewer adverse effects, especially renal effects, compared to indomethacin [9]. Persistent ductus arteriosus with moderate haemodynamic shunt is associated with lower respiratory tract infections, atelectasis or pneumonia. Newborns with large sized arterial channels and significant shunt at this level develop eating difficulties causing a deficient weight curve [4]. Symptoms of heart failure are a rare occurrence.
Ductal-dependent cardiac malformations require drug therapy for keeping the permeability of the ductus arteriosus until the time of corrective surgery $[10,11]$.

\section{CASE PRESENTATION}

We present the case of an extremely low birth weight premature new-born, admitted to the Regional Centre of Neonatal Intensive Care Unit Tîrgu-Mureş. The new-born had a gestational age of 31-32 weeks and was one of twins. Prenatal monitoring had been adequately carried out leading to a suspicion of pathologic development, feto-fetal transfusion syndrome and expected premature birth. The mother was healthy, G II, P II, $\mathrm{Rh}$ negative, without feto-maternal isoimmunization, or other significant medical history. The child was delivered by caesarean section due to severe intrauterine growth restriction secondary to feto-fetal transfusion syndrome and the severe discordance between foetuses.

The new-born weighed 1700 grams which conformed with intrauterine growth curves of between the $10^{\text {th }}$ and $90^{\text {th }}$ percentiles. The PC was $29 \mathrm{~cm}$ and the length was $41 \mathrm{~cm}$, having an Apgar Score of 8/1 min, 9/5 min respectively. Postpartum required resuscitation according to the accepted protocol and included upper airway clearance, tactile stimulation, lower airway clearance by laryngoscope blade and subsequent Neopuff positive pressure ventilation (PIP $25-30 \mathrm{~cm}$ $\mathrm{H}_{2} \mathrm{O}$, PEEP 5-6 cm $\mathrm{H}_{2} \mathrm{O}, \mathrm{FiO}_{2} 100 \%$ ). The new-born required admission to the Regional Centre of Neonatal Intensive Care Unit Tîrgu-Mureş, where the additional therapeutic measures were administered, namely late therapeutic administration of surfactant by the "Insure" method, invasive ventilatory support, cardiovascular support and associated antibiotherapy.

The development of a bilateral grade III intra-ventricular haemorrhage, with bilateral ventriculomegaly and intracranial hypertension phenomena required the implantation of a subcutaneous reservoir, followed by a ventriculo-peritoneal shunt. This resulted in a satisfactory postoperative result.

The second twin, a boy, had a weight of 950 grams which is below the 10th percentile of the intrauterine growth curves. The $\mathrm{PC}$ was $26 \mathrm{~cm}$, and the lenght $37 \mathrm{~cm}$, having an Apgar Score of 7/1min, 9/5min respectively. The determination of blood gases from the umbilical cord highlights were: $\mathrm{pH}$ 7.317, $\mathrm{pCO}_{2} 38.9$ $\mathrm{mmHg}, \mathrm{pO}_{2} 15.5 \mathrm{mmHg}, \mathrm{BE}-6.3 \mathrm{mmol} / \mathrm{L}, \mathrm{HCO}_{3} 20.1$ $\mathrm{mmol} / \mathrm{L}$. He was transferred to the Regional Centre of 
Neonatal Intensive Care Unit Tîrgu-Mureş and he was placed in a closed incubator to ensure thermal comfort. Treatment included cardiovascular monitoring by pulse oximetry, CPAP noninvasive pressional support (PEEP $4 \mathrm{~cm} \mathrm{H}_{2} 0 ; \mathrm{FiO}_{2} 21 \%$ ), appropriate antibiotic therapy together with peripheral intravenous infusion and trophic enteral alimentation. The development of an infectious episode manifested by an altered carbohydrate metabolism, digestive intolerance, thrombocytopenia, refractory metabolic acidosis and increased inflammatory reactants required a change in antibiotic therapy to match the sensitivity of the germ isolated from pharyngeal culture. The detection of a systolic murmur in the second week of life, the diastolic theft revealed by trans-fontanellar ultrasound as well as lowered diastolic blood pressure values raised the suspicion of a patent ductus arteriosus and therefore specific treatment with ibuprofen, a cyclooxygenase inhibitor, was initiated.

An echocardiography was carried out following progressive changes in the condition of the child and evidence of heart failure. This revealed a coarctation of the aorta below the emergence of the left subclavian artery. Taking into account that at the time of the examination, systemic circulation is not dependent on the presence of the ductus arteriosus, the initiation of Prostaglandin E1 therapy was not deemed necessary. Due to the decreased left ventricular contractility, dobutamine inotropic therapy was initiated $(5 \mu \mathrm{g} / \mathrm{kgc} / \mathrm{min})$ together with vasodilators and associated diuretic therapy.

Following the above prescribed medication the overall condition of both newborns gradually altered, with significant haemodynamic changes. Decreased flow in the abdominal aorta required the initiation of prostaglandin E1 treatment $(0.1 \mu \mathrm{g} / \mathrm{kgc} / \mathrm{min})$. Development of secondary dilated cardiomyopathy required further associated inotropic treatment, with the initiation of SIMV invasive ventilatory support (PIP: $18 \mathrm{~cm} \mathrm{H}_{2} \mathrm{O}$, PEEP: $5 \mathrm{~cm} \mathrm{H}_{2} \mathrm{O}, \mathrm{FiO}_{2}$ : 60\%, Ti: $\left.0.39 \mathrm{sec}\right)$. Monitoring and evaluation of blood gases demonstrated a gradual decrease of ventilation parameters. Following treatment with prostaglandin E1, aortic blood flow improved significantly, concurrently with improved ventricular contractility.

By the age of 2 months, a weight of 2000 grams was achieved, and at that time primary corrective surgery was performed. This involved resection of the coarctation area with termino-terminal anastomosis. The developing newborn presented a prematurity specific pathology, i.e. an aggressive form of retinopathy (in- traventricular haemorrhage grade I/II with subsequent resorption) which required correction by laser therapy,

At the age of 3 months the child was cardiorespiratory balanced and was being breast-fed.

This being so, the child was discharged with instructions to return for clinical and paraclinical re-evaluation in accordance with the normal follow-up schedule for risk newborns.

\section{DISCUSSION}

In our case, the feto-fetal transfusion syndrome suspicion with severe discordance between fetuses in a pregnant woman at risk of preterm birth imposed the birth by caesarean section in fetal interest. Although the first twin has an appropriate weight according to the intrauterine growth curves, the second twin shows severe intrauterine growth restriction. Both newborns developed prematurity specific pathology requiring hospitalization and treatment in the Regional Center of Neonatal Intensive Care Unit Tîrgu-Mureş. Even though in dichorionic mono amniotic pregnancies there are no haemodynamic differences between the larger and the smaller fetus, in pregnancies where feto-fetal transfusion syndrome is present the receiver fetus has higher aortic and pulmonary velocities than the donor fetus. In addition the frequency of cardiac malformations is about 2.5 times higher in the case of twin pregnancies complicated with pathology [12-14]. Therefore followup of twin monochorionic pregnancies requires special care in assessing the cardiovascular system $[15,16]$. In case of the presented newborn lack of specific secondary haematological changes, respectively association of anaemia-polycythaemia does not support the existence of a specific feto-fetal transfusion like syndrome, only intrauterine growth restriction of the donor fetus being present, with severe discordance between twins (above 30\%). The involvement of the renin-angiotensin system and the transfer of vasoactive substances from the donor fetus predispose the receiver fetus to the development of hypertension and cardiac remodelling through the occurrence of hypertensive cardiomyopathy [14]. Development of prematurity specific respiratory pathology resulted in a decreased pulmonary compliance that required therapeutic surfactant administration and pressional ventilatory support [2]. Low diastolic blood pressure values associated with the diastolic theft revealed by transfontanellar ultrasound in an extremely low birth weight premature newborn, 
without clinical evidence of ductal dependent cardiac malformation, raised the suspicion of a persistent ductus arteriosus. Taking into account the increased frequency of patent ductus arteriosus in extremely low birth weight premature newborns, as revealed by other studies [4,5], coupled with a respiratory tract infection, pathology common in infants with this malformation [4], initiation of cyclooxygenase inhibitor therapy was decided. Due to reduced renal side effects compared to other drugs, ibuprofen in three consecutive doses is administrated. Although current data are insufficient to support which primary therapeutic approach should be addressed first, surgical ligation or administration of inhibitors of cyclooxygenase, our preferred first approach is drug administration $[17,18]$.

Subsequent haemodynamic changes with decreased blood flow in the aorta required the initiation of prostaglandin E1 therapy for the reopening of the ductus arteriosus, it being necessary until the time of corrective surgery. Severe intrauterine growth restriction in the newborn has a multifactorial aetiology, both by the presence of feto-fetal transfusion syndrome but also by the existence of associated cardiac malformation. Even when it is echocardiographically diagnosed, it is difficult to assess the need to maintain the patency of the ductus arteriosus, as in the present case.

\section{CONCLUSION}

Although beneficial, prophylactic or therapeutic closure of a persistent ductus arteriosus may worsen the development of a new-born with a "silent "cardiac malformation and associated pathology. In case of extremely low birth weight premature newborns who develop specific complications and have an impaired development, the presence of a cardiac malformation must be suspected.

\section{-ACKNOWLEDGEMENTS}

We thank the Doctors from the Paediatric Cardiac Clinic and Paediatric Cardiovascular Surgery Clinic for their assistance.

\section{CONFLICT OF INTEREST}

The authors declare that they have no conflict of interest.

\section{REFERENCES}

1. Doshi AR, Syamasundar Rao P. Coarctation of Aorta-Management Options and Decision Making. Pediat Therapeut. 2012;S5:006.

2. Subramanian Siva KN, Barton Aimee M, Montazam Sepideh et al. Extremely Low Birth Weight Infant. Medscape Oct 2, 2012.

3. Mehta SK, Younoszai A, Pietz J, Achanti BP. Pharmacological closure of the patent ductus arteriosus. Images Paediatr Cardiol. 2003;5:1-15.

4. Kim LK, Milliken JC, Allen HD et al. Patent Ductus Arteriosus (PDA). Medscape Sep 24, 2014.

5. Mercanti I, Boubred F, Simeoni U. Therapeutic closure of the ductus arteriosus: benefits and limitations. J Matern Fetal Neonatal Med. 2009;22 Suppl 3:14-20.

6. Malhotra A, Sasi A, Miller SL, Jenkin G, Polglase GR. The Efficacy of Surfactant Replacement Therapy in the GrowthRestricted Preterm Infant: What is the Evidence? Front Pediatr. 2014;29:2:118.

7. Gudmundsdottir A, Johansson S, Håkansson S et al. Timing of Pharmacological Treatment for Patent Ductus Arteriosus and Risk of Secondary Surgery, Death or Bronchopulmonary Dysplasia: A Population-Based Cohort Study of Extremely Preterm Infants. Neonatology. 2014 Nov 18;107(2):87-92. [Epub ahead of print].

8. Mahony L, Carnero V, Brett C et al. Prophylactic Indomethacin Therapy for Patent Ductus Arteriosus in Very-Low-Birth-Weight Infants. Engl J Med. 1982;306:506-10.

9. Ohlsson A1, Walia R, Shah S. Ibuprofen for the treatment of patent ductus arteriosus in preterm and/or low birth weight infants. Cochrane Database Syst Rev. 2008 Jan 23.

10.Liberman RF, Getz KD, Lin AE. Delayed diagnosis of critical congenital heart defects: trends and associated factors. Pediatrics. 2014 Aug;134(2):e373-81.

11.Syamasundar Rao P. Coarctation of Aorta. Current Science Inc. Current Cardiology Reports. 2005;7:425-34.

12. Karatza AA, Wolfenden JL, Taylor MJO et al. Influence of twintwin transfusion syndrome on fetal cardiovascular structure and function: prospective case-control study of 136 monochorionic twin pregnancies. Heart. 2002;88:271-7.

13.Boom van den J, Battin M, Hornung T. Twin-twin transfusion syndrome, coarctation of the aorta and hypoplastic aortic arch: a case series report. Paediatr Child Health. 2010;46:76-9.

14. Cloherty JP. Multiple Birth, in Cloherty JP, Eichenwald EC, Stark AR (eds): Manual of Neonatal Care - Sixth edition. Lippincott Williams \& Wilkins, Philadelphia, 2007;87-93.

15.Al Ammouri I, Jaradat S, Radwan J. Severe coarctation of the aorta in a $900 \mathrm{~g}$ donor of twin-twin transfusion newborn with successful repeated transcatheter angioplasty: a case report. Cardiol Young. 2014; Apr 4:1-4. [Epub ahead of print].

16. Bahtiyar MO, Dulay AT, Weeks BP, Friedman AH, Copel JA. Prevalence of congenital heart defects in monochorionic/ diamniotic twin gestations: a systematic literature review. J Ultrasound Med. 2007;26:1491-8. 
32 - The Journal of Critical Care Medicine 2015;1(1)

17. Mosalli R, Alfaleh K. Prophylactic surgical ligation of patent ductus arteriosus for prevention of mortality and morbidity in extremely low birth weight infants. Cochrane Database Syst Rev. 2008 Jan 23.
Available online at: www.jccm.ro

18. Malviya M, Ohlsson A, Shah S. Surgical versus medical treatment with cyclooxygenase inhibitors for symptomatic patent ductus arteriosus in preterm infants. Cochrane Database Syst Rev. 2008 Jan 23. 